Bull. Korean Math. Soc. 52 (2015), No. 4, pp. 1133-1138

http://dx.doi.org/10.4134/BKMS.2015.52.4.1133

\title{
ON THE DIOPHANTINE EQUATION $(a n)^{x}+(b n)^{y}=(c n)^{z}$
}

\author{
Mi-Mi MA AND JiAn-Dong Wu
}

Abstract. In 1956, Jeśmanowicz conjectured that, for any positive integer $n$ and any primitive Pythagorean triple $(a, b, c)$ with $a^{2}+b^{2}=c^{2}$, the equation $(a n)^{x}+(b n)^{y}=(c n)^{z}$ has the unique solution $(x, y, z)=(2,2,2)$. In this paper, under some conditions, we prove the conjecture for the primitive Pythagorean triples $(a, b, c)=\left(4 k^{2}-1,4 k, 4 k^{2}+1\right)$.

\section{Introduction}

Let $n$ be a positive integer and let $(a, b, c)$ be a primitive Pythagorean triple such that $a^{2}+b^{2}=c^{2},(a, b, c)=1$ and $2 \mid b$. Clearly, the Diophantine equation

$$
(n a)^{x}+(n b)^{y}=(n c)^{z}
$$

has the solution $(x, y, z)=(2,2,2)$. In 1956, Sierpiński [10] showed there is no other solutions when $n=1$ and $(a, b, c)=(3,4,5)$, and Jeśmanowicz [4] further proved that when $n=1$ and $(a, b, c)=(5,12,13),(7,24,25),(9,40,41)$, $(11,60,61)$, then $(1.1)$ has only the solution $(x, y, z)=(2,2,2)$. Moreover, he conjectured that for any positive integer $n,(1.1)$ has the unique solution $(x, y, z)=(2,2,2)$. Since then, many special cases of Jeśmanowicz' conjecture have been solved for $n=1$. In 1959, Lu [8] proved the conjecture when $n=1$ and $(a, b, c)=\left(4 k^{2}-1,4 k, 4 k^{2}+1\right)$. Later, Deḿjanenko [1] verified the conjecture if $n=1$ and $c=b+1$. Recently, Miyazaki [9] showed that Jeśmanowicz' conjecture is true if $n=1$ and $a \equiv \pm 1(\bmod b)$ or $c \equiv 1(\bmod b)$. This result generalized the results of $\mathrm{Lu}$ and Deḿjanenko. For more results, see $[5,6,11,12,13,15]$.

When $n>1$, only a few results on this conjecture are known. For any positive integer $t$ with $t>1$, let $P(t)$ denote the product of distinct prime factors of $t$. In 1998, Deng and Cohen [3] proved that if $n>1, c=b+1, a$ is a prime power and either $P(b) \mid n$ or $P(n) \nmid b$, then (1.1) has only the solution

Received May 16, 2014; Revised December 30, 2014.

2010 Mathematics Subject Classification. 11D61.

Key words and phrases. Jeśmanowicz' conjecture, Diophantine equation, Pythagorean triple.

This work is supported by National Natural Science Foundation of China (Grant No. 11371195 and 11401411) and Program of Natural Science Research of Jiangsu Higher Education Institutions of China (Grant No. 13KJD110005). 
$(x, y, z)=(2,2,2)$. In 1999, Le [7] gave certain necessary conditions for $(1.2)$ to have positive integer solutions $(x, y, z)$ with $(x, y, z) \neq(2,2,2)$.

Recently, some special cases of the Pythagorean triple $(a, b, c)=\left(4 k^{2}-\right.$ $\left.1,4 k, 4 k^{2}+1\right)$ have been considered. Then (1.1) becomes

$$
\left(n\left(4 k^{2}-1\right)\right)^{x}+(4 n k)^{y}=\left(n\left(4 k^{2}+1\right)\right)^{z} .
$$

For instance, Yang and Tang [16] proved that Jeśmanowicz' conjecture is true when $k=2$. Tang and Weng [14] proved that Jeśmanowicz' conjecture is true for $(a, b, c)=\left(F_{k}-2,2^{2^{k-1}+1}, F_{k}\right)$, where $F_{k}=2^{2^{k}}+1$ is $k$-th Fermat number. Deng [2] proved the following results: (1) For $k=2^{s}(1 \leq s \leq 4)$ and any positive integer $n$, the only solution of $(1.2)$ is $(x, y, z)=(2,2,2)$; (2) For $k=2^{s}(s \geq 0)$ and any positive integer $n$ with $P(a) \mid n$ or $P(n) \nmid a$, the only solution of $(1.2)$ is $(x, y, z)=(2,2,2)$.

In this paper, we always assume that $(a, b, c)=\left(4 k^{2}-1,4 k, 4 k^{2}+1\right)$. The following results are proved.

Theorem 1.1. Suppose that the positive integer $n$ is such that $P(a) \mid n$. Then the only solution of $(1.2)$ is $(x, y, z)=(2,2,2)$.

Theorem 1.2. Let $k=p^{\alpha}$, where $\alpha \geq 0$ and $p$ is a prime with $p \equiv-1$ $(\bmod 4)$. Suppose that $n$ is a positive integer with $P(n) \nmid a$. Then the only solution of $(1.2)$ is $(x, y, z)=(2,2,2)$.

\section{Lemmas}

Lemma 2.1 ([8, Theorem $])$. Let $n=1$. Then (1.2) has the only positive integer solution $(x, y, z)=(2,2,2)$.

Lemma 2.2 ([2, Corollary 2.4]). Let $(a, b, c)$ be any primitive Pythagorean triple such that the Diophantine equation $a^{x}+b^{y}=c^{z}$ has the only positive integer solution $(x, y, z)=(2,2,2)$. If $(x, y, z)$ is a solution of (1.2) with $(x, y, z) \neq(2,2,2)$, then one of the following conditions is satisfied:

(1) $x>z>y$ and $P(n) \mid b$

(2) $y>z>x$ and $P(n) \mid a$.

Lemma 2.3. Suppose that the positive integer $n$ is such that $P(n) \mid b$. If $(x, y, z)$ is a solution of $(1.2)$ with $(x, y, z) \neq(2,2,2)$ and $2 \mid z$, then $\frac{b^{y}}{n^{z-y}}$ is not a square number.

Proof. By Lemma 2.2, we have $x>z>y$. Assume that $\frac{b^{y}}{n^{z-y}}=d^{2}$ is a square number. By (1.2), we have

$$
\frac{b^{y}}{n^{z-y}}=c^{z}-a^{x} \cdot n^{x-z} .
$$

Write $z=2 z_{1}$. Then

$$
a^{x} \cdot n^{x-z}=c^{z}-d^{2}=\left(c^{z_{1}}-d\right)\left(c^{z_{1}}+d\right) .
$$


Noting that $a$ is odd and

$$
\left(c^{z_{1}}-d, c^{z_{1}}+d\right) \leq\left(2 c^{z_{1}}, 2 d\right) \leq\left(2 c^{z_{1}}, 2 b^{y}\right)=2,
$$

we have $a=a_{1} a_{2}$ with $\operatorname{gcd}\left(a_{1}, a_{2}\right)=1, a_{1}^{x} \mid c^{z_{1}}+d$ and $a_{2}^{x} \mid c^{z_{1}}-d$. Let $a_{i}=\max \left\{a_{1}, a_{2}\right\}$. By $a_{1} a_{2}=a=(2 k-1)(2 k+1)$, we have $a_{i} \geq 2 k+1$. Thus

$$
\begin{aligned}
a_{i}^{x} & >\left(a_{i}^{2}\right)^{z_{1}} \geq\left(4 k^{2}+1+4 k\right)^{z_{1}}=(c+b)^{z_{1}} \\
& \geq c^{z_{1}}+b^{z_{1}}>c^{z_{1}}+b^{\frac{y}{2}} \geq c^{z_{1}}+d>c^{z_{1}}-d,
\end{aligned}
$$

a contradiction.

\section{Proof of Theorem 1.1}

Since $P(a) \mid n$ and $(a, b)=1$, it follows that $P(n) \nmid b$. By Lemma 2.2, we have $y>z>x$ and $P(n) \mid a$. Thus $P(n)=P(a)$. By (1.2), we have

$$
a^{x}=n^{z-x}\left(c^{z}-b^{y} \cdot n^{y-z}\right) .
$$

By $P(n)=P(a)$, we have $n^{z-x}=a^{x}$. It follows that

$$
b^{y} \cdot n^{y-z}=c^{z}-1 .
$$

Let $k=2^{l} \cdot k_{1}, 2 \nmid k_{1}$. Noting that $a=4 k^{2}-1, b=4 k, c=4 k^{2}+1$, we have

$\left(2^{l+2} \cdot k_{1}\right)^{y} \cdot n^{y-z}=\left(2^{2 l+2} \cdot k_{1}^{2}+1\right)^{z}-1=z \cdot 2^{2 l+2} \cdot k_{1}^{2}+\frac{z(z-1)}{2}\left(2^{2 l+2} \cdot k_{1}^{2}\right)^{2}+\cdots$.

By $x<z<y$, we have $y \geq 2$. The power of 2 in $\left(2^{l+2} \cdot k_{1}\right)^{y} \cdot n^{y-z}$ is $(l+2) y \geq 2 l+4$. By (3.3), we have $2 \mid z$. Write $z=2 z_{1}$. It follows that

$$
b^{y} \cdot n^{y-z}=\left(c^{z_{1}}-1\right)\left(c^{z_{1}}+1\right) .
$$

Note that $\left(c^{z_{1}}+1, b^{y}\right)=2$, we have $\frac{b^{y}}{2} \mid c^{z_{1}}-1$. However,

$$
\frac{b^{y}}{2}>\frac{b^{2 z_{1}}}{2}=\frac{(c-a)^{z_{1}}(c+a)^{z_{1}}}{2} \geq c^{z_{1}}+a^{z_{1}}>c^{z_{1}}-1
$$

a contradiction.

\section{Proof of Theorem 1.2}

We suppose that $(1.2)$ has a solution $(x, y, z) \neq(2,2,2)$, and will observe that this leads to a contradiction. By Lemma 2.1, we may assume that $n \geq 2$.

By $P(n) \nmid a$ and Lemma 2.2, we have $y<z<x$ and $P(n) \mid b$. Write $n=2^{r} \cdot p^{s}$, where $r+s \geq 1$. By (1.2), we have

$$
b^{y}=n^{z-y}\left(c^{z}-a^{x} \cdot n^{x-z}\right),
$$

or equally

(4.2) $2^{2 y} \cdot p^{\alpha y}=2^{r(z-y)} \cdot p^{s(z-y)}\left(\left(4 \cdot p^{2 \alpha}+1\right)^{z}-\left(4 \cdot p^{2 \alpha}-1\right)^{x} \cdot 2^{r(x-z)} \cdot p^{s(x-z)}\right)$.

Case 1. $r \geq 1, s=0$. Then $n=2^{r}$. By (4.2),

$$
2^{2 y} \cdot p^{\alpha y}=2^{r(z-y)}\left(\left(4 \cdot p^{2 \alpha}+1\right)^{z}-\left(4 \cdot p^{2 \alpha}-1\right)^{x} \cdot 2^{r(x-z)}\right) .
$$


So $2 y=r(z-y)$ and

$$
p^{\alpha y}=\left(4 \cdot p^{2 \alpha}+1\right)^{z}-\left(4 \cdot p^{2 \alpha}-1\right)^{x} \cdot 2^{r(x-z)} .
$$

If $r(x-z) \geq 3$, by (4.3), we have $(-1)^{\alpha y} \equiv 1(\bmod 4)$. Thus $2 \mid \alpha y$. Also by (4.3), we have $5^{z} \equiv 1(\bmod 8)$. Hence $2 \mid z$. By Lemma 2.3 , this is impossible. Then $r(x-z)=1$ or 2 . If $\alpha y \geq 2$, then by (4.3), we have $(-1)^{x} \cdot 2^{r(x-z)} \equiv 1$ $\left(\bmod p^{2}\right)$. Note that $p \geq 3$, this is impossible. Then $\alpha y=1$. We have $\alpha=1, y=1$. It follows that

$$
\left(4 \cdot p^{2}-1\right)^{x} \cdot 2^{r(x-z)}=\left(4 \cdot p^{2}+1\right)^{z}-p .
$$

We have $(-1)^{x} \cdot 2^{r(x-z)} \equiv 1(\bmod p)$. Note that $r(x-z)=1,2$ and $p \equiv-1$ $(\bmod 4)$, we have $p=3$. By $(4.4)$, we get

$$
35^{x} \cdot 2^{r(x-z)}=37^{z}-3 .
$$

If $r(x-z)=1$, then $r=1, x-z=1$. Note that $2 y=r(z-y)$ and $y=1$, we have $z=3 y=3$ and $x=4$. Then $3001250=35^{4} \cdot 2=37^{3}-3=50650$, this is impossible.

If $r(x-z)=2$, then $r=2, x-z=1$ or $r=1, x-z=2$. If $r=2, x-z=1$, then we have $z=2 y=2$ and $x=3$. By (4.5), we have $171500=35^{3} \cdot 2^{2}=$ $37^{2}-3=1366$, this is impossible. If $r=1, x-z=2$, we have $z=3 y=3$ and $x=5$. It follows that $35^{5} \cdot 2^{2}=37^{3}-3$, this is impossible.

Case 2. $r=0, s \geq 1$. Then $n=p^{s}$. By (4.2),

$$
2^{2 y} \cdot p^{\alpha y}=p^{s(z-y)}\left(\left(4 \cdot p^{2 \alpha}+1\right)^{z}-\left(4 \cdot p^{2 \alpha}-1\right)^{x} \cdot p^{s(x-z)}\right) .
$$

So $\alpha y=s(z-y)$ and

$$
2^{2 y}=\left(4 \cdot p^{2 \alpha}+1\right)^{z}-\left(4 \cdot p^{2 \alpha}-1\right)^{x} \cdot p^{s(x-z)} .
$$

By (4.6), we have $(-1)^{x+s(x-z)} \equiv 1(\bmod 4)$. Then $2 \mid x+s(x-z)$. If $y=1$, then

$$
4=\left(4 \cdot p^{2 \alpha}+1\right)^{z}-\left(4 \cdot p^{2 \alpha}-1\right)^{x} \cdot p^{s(x-z)} .
$$

By $(4.7)$, we have $4 \equiv 1(\bmod p)$, so $p=3$. We have $5^{z}-3^{x+s(x-z)} \equiv 5^{z}-1 \equiv 4$ $(\bmod 8)$, so $2 \nmid z$. Note that $\alpha y=s(z-y)$, we have $\alpha=s(z-1)$, so $2 \mid \alpha$. By (4.7), we have $2^{z} \equiv 4\left(\bmod 2 \cdot 3^{\alpha}+1\right)$. Let $\left(\frac{*}{*}\right)$ denote the Jacobi symbol. Note that $2 \cdot 3^{\alpha}+1 \equiv 3(\bmod 8)$, we have

$$
-1=\left(\frac{2}{2 \cdot 3^{\alpha}+1}\right)=\left(\frac{2}{2 \cdot 3^{\alpha}+1}\right)^{z}=\left(\frac{4}{2 \cdot 3^{\alpha}+1}\right)=1,
$$

a contradiction. So $y \geq 2$. If $p=3$, then, by (4.6), we have $5^{z} \equiv 3^{x+s(x-z)} \equiv 1$ $(\bmod 8)$. Thus $2 \mid z$. By Lemma 2.3 , this is impossible. If $p>3$, by (4.6), we have $(-1)^{z} \equiv 1(\bmod 3)$. So $2 \mid z$. By Lemma 2.3 , this is impossible.

Case 3. $r \geq 1, s \geq 1$. It is obviously that $2 p \nmid\left(4 \cdot p^{2 \alpha}+1\right)^{z}-\left(4 \cdot p^{2 \alpha}-1\right)^{x}$. $2^{r(x-z)} \cdot p^{s(x-z)}$. By $(4.2)$, we have $2 y=r(z-y), \alpha y=s(z-y)$ and

$$
1=\left(4 \cdot p^{2 \alpha}+1\right)^{z}-\left(4 \cdot p^{2 \alpha}-1\right)^{x} \cdot 2^{r(x-z)} \cdot p^{s(x-z)},
$$


or equally

$$
\left(4 \cdot p^{2 \alpha}-1\right)^{x} \cdot 2^{r(x-z)} \cdot p^{s(x-z)}=\left(4 \cdot p^{2 \alpha}+1\right)^{z}-1 .
$$

So $\alpha r=2 s$. Note that $p^{2 \alpha} \mid\left(4 \cdot p^{2 \alpha}+1\right)^{z}-1$, we have $s(x-z) \geq 2 \alpha$. It turns out that $r s(x-z) \geq 2 \alpha r=4 s$, i.e., $r(x-z) \geq 4$. By (4.8), we have $5^{z} \equiv 1$ $(\bmod 8)$, so $2 \mid z$. By Lemma 2.3 , this is impossible.

This completes the proof of Theorem 1.2.

Acknowledgments. We sincerely thank our supervisor Professor Yong-Gao Chen for his valuable suggestions and useful discussions.

\section{References}

[1] Deḿjanenko, On Jeśmanowicz' problem for Pythagorean numbers, Izv. Vyss. Ucebn. Zaved. Mat. 48 (1965), no. 5, 52-56.

[2] M. J. Deng, A note on the Diophantine equation $(n a)^{x}+(n b)^{y}=(n c)^{z}$, Bull. Austral. Math. Soc. 89 (2014), no. 2, 316-321.

[3] M. J. Deng and G. L. Cohen, On the conjecture of Jeśmanowicz concerning Pythagorean triples, Bull. Austral. Math. Soc. 57 (1998), no. 3, 515-524.

[4] L. Jeśmanowicz, Several remarks on Pythagorean numbers, Wiadom. Mat. 1 (1955/56), 196-202.

[5] M. H. Le, A note on Jeśmanowicz conjecture, Colloq. Math. 69 (1995), no. 1, 47-51.

[6] , On Jeśmanowicz conjecture concerning Pythagorean numbers, Proc. Japan Acad. Ser. A Math. Sci. 72 (1996), no. 5, 97-98.

[7] _ A note on Jeśmanowicz' conjecture concerning Pythangorean triples, Bull. Austral. Math. Soc. 59 (1999), no. 3, 477-480.

[8] W. T. Lu, On the Pythagorean numbers $4 n^{2}-1,4 n$ and $4 n^{2}+1$, Acta Sci. Natur. Univ. Szechuan 2 (1959), 39-42.

[9] T. Miyazaki, Generalizatioins of classical results on Jeśmanowicz' conjecture concerning Pythagorean triples, J. Number Theory 133 (2013), no. 2, 583-595.

[10] W. Sierpiński, On the Diophantine equation $3^{x}+4^{y}=5^{z}$, Wiadom. Mat. 1 (1955/56), 194-195.

[11] K. Takakuwa, On a conjecture on Pythagorean numbers. III, Proc. Japan Acad. Ser. A Math. Sci. 69 (1993), no. 9, 345-349.

[12] $\longrightarrow$ A remark on Jeśmanowicz' conjecture, Proc. Japan Acad. Ser. A Math. Sci. 72 (1996), no. 6, 109-110.

[13] K. Takakuwa and Y. Asaeda, On a conjecture on Pythagorean numbers. II, Proc. Japan Acad. Ser. A Math. Sci. 69 (1993), no. 8, 287-290.

[14] M. Tang and J. X. Weng, Jeśmanowicz' conjecture with Fermat numbers, Taiwanese J. Math. 18 (2014), no. 3, 925-930.

[15] M. Tang and Z. J. Yang, Jeśmanowicz' conjecture revisited, Bull. Austral. Math. Soc. 88 (2013), no. 3, 486-491.

[16] Z. J. Yang and M. Tang, On the Diophantine equation $(8 n)^{x}+(15 n)^{y}=(17 n)^{z}$, Bull. Austral. Math. Soc. 86 (2012), no. 2, 348-352.

Mi-Mi MA

School of Mathematical Sciences and Institute of Mathematics

NANJing NORMAL University

NANJing 210023, P. R. China

E-mail address: 602640993@qq.com 
JiAn-DONG WU

School of Mathematical Sciences and Institute of Mathematics

NANJing Normal University

NANJing 210023, P. R. ChinA

E-mail address: wjd.njnu@163.com 